\title{
Hope and Mood Improvement in Women with Breast Cancer Using Group Poetry Therapy: A Questionnaire-based Before-After Study
}

\author{
Ghasem Janbabai ${ }^{1}$, Pouran Daboui ${ }^{2}$, Siavash Moradi ${ }^{3,}$ \\ ${ }^{1}$ Department of Medical Oncology, Gastrointestinal Cancer Research Center, \\ Mazandaran University of Medical Sciences, Sari, Iran \\ ${ }^{2}$ Department of Persian Literature, Faculty of Human Sciences, Islamic Azad \\ University, Central Tehran Branch, Tehran, Iran \\ ${ }^{3}$ Department of Community and Preventive Medicine, Gastrointestinal Cancer \\ Research Center, Mazandaran University of Medical Sciences, Sari, Iran
}

* Corresponding author: Siavash Moradi, Department of Community and Preventive Medicine, Gastrointestinal Cancer Research Center, Mazandaran University of

DOI: $10.21859 /$ mci-supp-95 Medical Sciences, Sari, Iran.E-mail:d.smor86@yahoo.com

\section{Keywords:}

Cancer

Poetry Therapy

Questionnaire

Hope

Mood

\begin{abstract}
Introduction: Cancer is one of the most challenging diseases and patients are rarely able to maintain their mental health when dealing with it. This study seeks to use the great works of group poetry therapy as a supportive device for improving mental health and increasing hope in patients with breast cancer.

Material and Methods: This before-after study was conducted in 2016 on women with breast cancer undergoing chemotherapy at a referral center at the north of Iran. The study protocol included eight weekly sessions of group poetry therapy using poems of great Persian poets. The patients completed two questionnaires, including Miller's Hope Scale (MHS) and Depression Anxiety Stress Scales (DASS-21) before beginning group poetry therapy and again one week and two months after the sessions had ended. Items of each questionnaire were manually scored and then analyzed using IBM SPSS-21.

Results: 29 patients participated in all the group poetry therapy sessions, all 29 completed DASS-21, and 28 completed MHS. Increase in the hope score and reduction in the stress score were observed in two-month follow-up and both were statistically significant ( $\mathrm{p}=$ 0.01 and 0.02 respectively).

Conclusions: This study suggests that group poetry therapy can improve moods and hopefulness in women with breast cancer. Also the formation of a small group of patients who use the mystical themes of Persian poetry to connect to each other can have lasting positive effects in the long term.
\end{abstract}

